


\section{Diseño de estrategias para el mejoramiento continuo de la calidad en el proceso de producción de productos de panadería de una microempresa}

Keren Inés Barboza Lunaㄹ, Camilo Fabián Cáceres Mercado², Sandra Marcela Figueroa Alvis ${ }^{3}$, Diego Andrés Mayoriano Contreras ${ }^{4}$

\section{Resumen}

La calidad de los bienes de servicios depende de la gestión de varios factores, dentro de los cuales podemos mencionar el control de las operaciones que dan como resultado dichos bienes y servicios. Este control operacional puede hacerse a través del control estadístico de procesos, el cual utiliza una serie de métodos y procedimientos que permiten el monitoreo y análisis de los procesos, con el fin de lograr su estabilidad y mejorar la capacidad de los mismos en términos de cumplir con las especificaciones de los productos fabricados. A pesar de la importancia del control estadístico de procesos, es posible afirmar que son pocas las micro y pequeñas empresas que aplican estos métodos en el departamento de Sucre, por lo cual estas desconocen el comportamiento estadístico de sus procesos, lo que impide una adecuada toma de decisiones en aras de su mejoramiento continuo. Si una empresa no mejora continuamente los procesos se aleja del camino de la calidad, lo cual repercute negativamente en la productividad y competitividad de la misma. En consecuencia, con lo anteriormente expuesto, se realiza este estudio con el ánimo de contribuir en la búsqueda de la calidad por parte de la microempresa

1 Ingeniera Industrial. Corporación Universitaria del Caribe-CECAR. Correo: keren.barboza@cecar.edu.co; Orcid: https://orcid.org/0000-0001-8998-8112 2 Ingeniero Industrial. Corporación Universitaria del Caribe-CECAR. Correo: camilo.caceres@cecar.edu.co; Orcid: https://orcid.org/0000-0002-5077-7331 3 Ingeniera Industrial. Corporación Universitaria del Caribe-CECAR. Correo: sandra.figueroa@cecar.edu.co; Orcid: https://orcid.org/0000-0001-8382-1266 4 Ingeniero Industrial. Corporación Universitaria del Caribe-CECAR. Correo: diego.mayoriano@cecar.edu.co; Orcid: https://orcid.org/0000-0001-5820-6800 
considerada para el mismo. Las aplicaciones de estas metodologías permitieron identificar los principales problemas de calidad de la empresa y diseñar estrategias para la eliminación de las causas principales de los mismos, entre las cuales podemos mencionar: estandarizar el proceso de medición de la cantidad de ingredientes a utilizar, establecer programas de capacitación y la adquisición de herramientas de corte de mayor precisión.

Palabras clave: cartas de control, pruebas de normalidad, diagrama de causa-efecto, calidad, matriz AMEF.

\section{Design of strategies for continuous quality improvement in the production process of bakery products of a microenterprise}

\section{Abstract}

The quality of the goods of services depends on the management of several factors, within which we can mention the control of the operations that result in said goods and services. This operational control can be done through the statistical control of processes which uses a series of methods and procedures that allow the monitoring and analysis of the processes in order to achieve their stability and improve their capacity in terms of complying with the specifications of the products manufactured. Despite the importance of statistical process control, it is possible to affirm that there are few micro and small companies that apply these methods in the department of Sucre, so they are unaware of the statistical behavior of their processes, which prevents adequate decision-making. for the continuous improvement of processes. If a company does not continuously improve processes, it moves away from the path of quality, which negatively affects its productivity and competitiveness. Consequently, with the above, this study is carried out with the aim of contributing to the search for quality by the micro-company considered for it. The applications of these methodologies allowed to identify the main problems of quality of the company and to design strategies for the elimination of the main causes of the same

Keywords: control charts, normality tests, cause-effect diagram, quality, AMEF matrix. 


\section{Introducción}

Hoy en día, la calidad es fundamental para cualquier empresa pues muestra que los procesos están marchando correctamente y que se están obteniendo productos de excelencia: el control estadístico de la calidad es una pieza clave para la obtención de esta. Asimismo, aplicar control de la calidad a todos los procesos de la empresa permite mejorar de forma continua la organización, aumentando la rentabilidad del negocio y el grado de satisfacción del cliente (Gutiérrez, 2009). Es por esto que el presente estudio tiene como objetivo evaluar el proceso de pesaje de tres tipos de panes, en donde la variable que se pretende estudiar es la siguiente:

X: "Peso en gramos del pan ocañero, pan integral y pan francés"

La justificación de la variable seleccionada radica en que los panaderos no utilizan instrumentos de medición para el pesaje del pan, debido a que la panadería no cuenta con dichas herramientas, motivo por el cual el tamaño del pan es variable. La realización del presente estudio obedece a motivos económicos, dado que la aplicación de estrategias que eviten los problemas que se encuentran en el proceso del pesaje del pan permitirá reducir costes y maximizar beneficios. En este sentido, el objetivo del estudio es observar la variación del tamaño de cada pan, teniendo en cuenta el valor nominal y la tolerancia establecida en la panificadora; para ello se utilizan herramientas de control de calidad como histogramas, pruebas de normalidad, cartas de control, entre otras. Posteriormente se muestran los resultados obtenidos, las conclusiones y recomendaciones.

\section{Materiales y métodos}

Para la recolección de información se realizó la medición del peso de los panes (pan ocañero, pan integral, pan francés) en gramos. Para ello, se tomaron 4 muestras de tamaño 36 para el pan ocañero y pan integral; y de tamaño 45, para el pan francés. A continuación, se muestra la tabla 1 , la cual indica el valor nominal y la tolerancia del peso neto de cada pan. Posteriormente, en la tabla 2 se muestra la media y la desviación estándar del peso neto de los tres panes objeto de estudio. De la tabla 2 se puede 
inferir que el pan que presenta mayor dispersión de datos con respecto a la media es el pan ocañero.

\section{Tabla 1}

Valor nominal y tolerancia del peso neto $(\mathrm{gr})$ de los panes que son objeto de estudio.

\begin{tabular}{llll}
\hline & Pan ocañero & Pan integral & Pan francés \\
\hline Nominal & 28 & 75 & 80 \\
Tolerancia & 2 & 3 & 3 \\
Total datos & 36 & 72 & 45 \\
\hline
\end{tabular}

Nota. Fuente: construcción propia.

Tabla 2

Datos de tendencia central del peso neto de los panes.

\begin{tabular}{lccc}
\hline $\begin{array}{c}\text { Tipos de pan / medidas de } \\
\text { tendencia central }\end{array}$ & $\begin{array}{c}\text { Pan } \\
\text { ocañero }\end{array}$ & $\begin{array}{c}\text { Pan } \\
\text { integral }\end{array}$ & Pan francés \\
\hline Media & 28,44 & 81,23 & 74,55 \\
Desviación estándar & 1,58 & 0,75 & 0,82 \\
\hline
\end{tabular}

Nota. Fuente: construcción propia

Luego de obtener los datos necesarios para realizar el estudio de calidad, se hicieron un histograma y pruebas de normalidad con el fin de observar, de forma gráfica, la distribución de peso de los panes para comprobar que los datos tuvieran un comportamiento normal (Cartín et al., 2014). Para los cálculos y la elaboración del histograma de frecuencia fue necesario tomar la primera muestra del peso de los panes y su respectivo valor nominal, estos histogramas fueron realizados en los softwares Minitab y Excel (Rubio, Angeles, Soto, \& Toma, 1995). Para su elaboración en Excel se procedió a efectuar los siguientes pasos:

1) Se determinó el máximo y el mínimo de los datos.

2) Se calculó el rango

$\mathrm{R}=\mathrm{X} \max -\mathrm{X} \min (1)$

3) Se calculó el número de clases

$\mathrm{m}=1+3,3 * \log (\mathrm{n})(2)$

4) Se calculó la amplitud de clase 
5) Se determinaron las clases y las frecuencias de clase.

$$
C=\frac{\text { Rango }}{\text { número de clases }(m)}
$$

Para probar que los datos tuvieran una distribución normal, se realizó el test de Kolmogorov. Este indicador se aplica con el fin de probar la hipótesis de normalidad de la población que se estudia; para su realización se ejecutaron los siguientes pasos:

1) Se creó una columna con un consecutivo que va desde 1 hasta el número del tamaño de la muestra.

2) Se creó una columna con los datos ordenados de menor a mayor.

3) Se calculó el cociente

4) Se calculó la probabilidad de que se asuman valores menores o iguales a cada uno de los datos muestrales, con la siguiente función de Excel

distr.norm.n(dato muestral;media;desv_estandar;1)

5) Se halló la diferencia entre la columna del cociente y la de la probabilidad

\section{diferencia=cociente-probabilidad (5)}

6) Se determinaron los valores absolutos de la diferencia y el valor mayor.

7) Se comparó el mayor valor hallado (mayor valor absoluto) en el paso anterior con el valor correspondiente a la tabla.

Luego de comprobar que los datos se distribuyen de forma normal, se aplican las cartas de control, con la finalidad de detectar variabilidad en el proceso (Rivera García, 2011); esta herramienta permite observar si el proceso se encuentra bajo control estadístico (Gutiérrez, 2009). Para su aplicación, se procedió a efectuar los siguientes cálculos:

1) Se recolectaron las cuatro muestras.

2) Se calculó la media para cada subgrupo mediante la siguiente ecuación: 


$$
\bar{x}=\sum_{i=1}^{n} x_{i} / n
$$

Donde $\mathrm{n}=$ tamaño de cada subgrupo

3) Se calculó la media general

$$
\overline{\bar{x}}=\sum_{i=1}^{k} \bar{x}_{i} / k
$$

Donde $k$ es el número de subgrupos y $\bar{x}_{i}$ es la media de cada subgrupo.

4) Se calculó el rango

$$
R=X_{\max }-X_{\min }
$$

5) Se calculó $\bar{R}$ : para ello se calculó la media del rango

$$
\bar{R}=\sum_{i=1}^{k} R_{i} / \mathrm{k}
$$

6) Se calcularon las líneas de control teniendo en cuenta una nueva media y desviación estándar (Rojas, 2006)

$$
\begin{gathered}
\mu=\overline{\bar{X}} \\
\sigma=\frac{\bar{R}}{d_{2}}
\end{gathered}
$$

Límite de control superior $(\mathrm{LCS})=\overline{\overline{\mathrm{X}}}+\mathrm{A}_{2} * \overline{\mathrm{R}}$ (12)

$$
\text { Línea central }(\mathrm{LC})=\overline{\overline{\mathrm{x}}}(13)
$$

Límite de control inferior (LCI) $=\overline{\overline{\mathrm{x}}}-\mathrm{A}_{2} * \overline{\mathrm{R}}$ (14)

Luego, se determinaron los índices de capacidad de proceso (Mosquera, Artamonova, \& Mosquera, 2013), con el propósito de evaluar si el proceso es capaz de cumplir con las especificaciones deseadas. Para esto se tomaron los datos ya descritos anteriormente y el intervalo de 
tolerancia de cada uno de los panes. Las respectivas ecuaciones empleadas para determinar los índices de capacidad son las siguientes:

$$
\begin{gathered}
c p=\frac{\text { LES-LEI }}{6 * \sigma} \\
C_{p S}=\frac{L S E-\mu}{3 * \sigma} \\
C_{p i}=\frac{\mu-L I E}{3 * \sigma}
\end{gathered}
$$

Posteriormente, se determinó el porcentaje de productos que se encuentran por fuera de los límites de especificación, es decir, productos defectuosos. El análisis de estos defectos fue realizado a través de un diagrama causa y efecto; este diagrama permite obtener un cuadro, detallado y de fácil visualización, de las diversas causas que pueden originar un determinado efecto o problema. Además, se realizó una matriz AMEF con el fin de analizar las fallas del proceso de pesaje de los panes, para así evaluar y clasificar sus efectos con el fin de evitar su ocurrencia (Cartín, Villarreal, \& Morera, 2014). 


\section{Resultados y discusión}

Por otra parte, para observar la distribución de frecuencia del peso neto de cada pan se realizó un histograma múltiple, el cual se muestra en la figura 1 .

Figura 1

Histograma para cada tipo de pan.

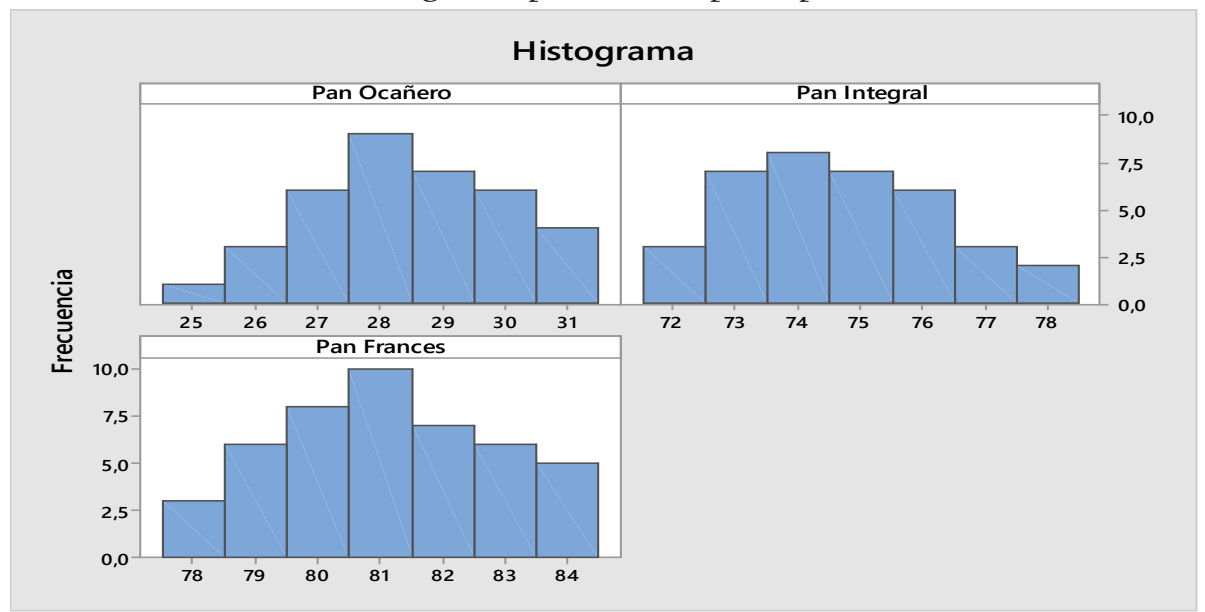

Nota. Fuente: construcción propia

Los anteriores histogramas muestran que los datos se aproximan a una distribución normal. No obstante, se puede observar que el histograma del pan integral se encuentra sesgado a la derecha (sesgo positivo). Del anterior resultado, se puede inferir que el proceso que se realiza está operando bajo los estándares o condiciones de normalidad. Cabe destacar que algunos datos se encuentran por fuera de los límites de especificación (inferior y superior), como se muestra en la figura 2. 


\section{Figura 2}

Límites de especificación para cada tipo de pan.

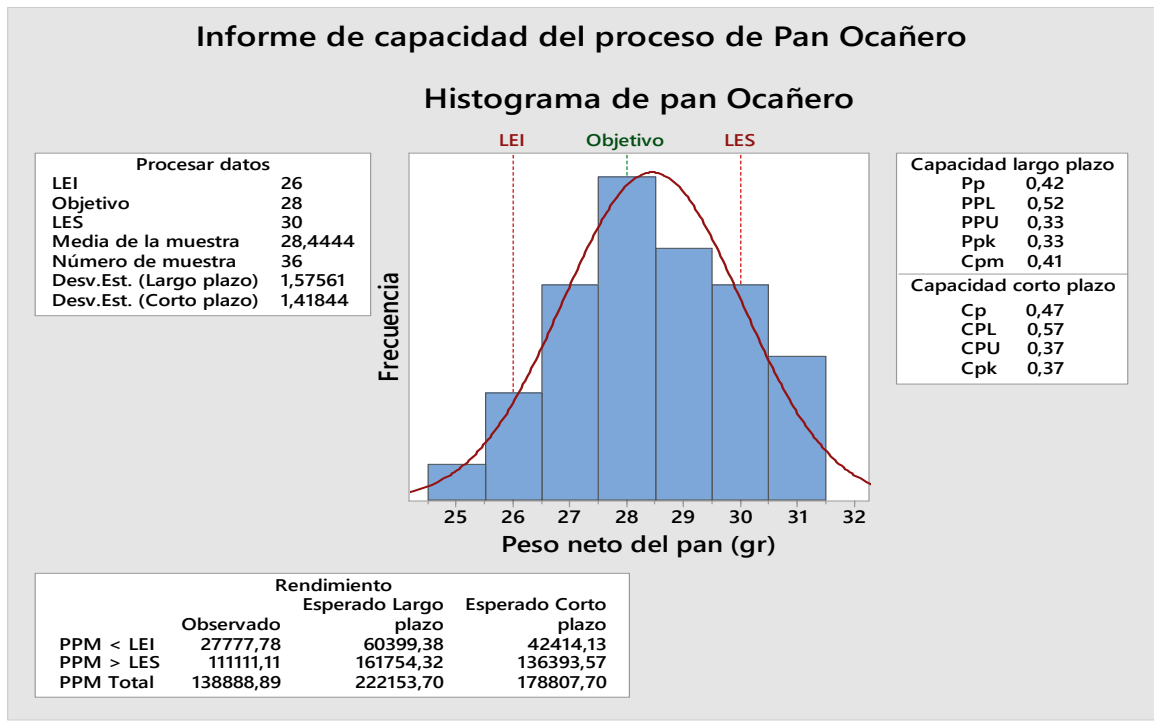

\section{Informe de capacidad del proceso de Pan Integral}

Histograma de pan Integral

\begin{tabular}{|ll|}
\hline \multicolumn{2}{|c|}{ Procesar datos } \\
LEI & 72 \\
Objetivo & 75 \\
LES & 78 \\
Media de la muestra & 74,6389 \\
Número de muestra & 36 \\
Desv.Est. (Largo plazo) & 1,64148 \\
Desv.Est. (Corto plazo) & 1,69706 \\
\hline
\end{tabular}

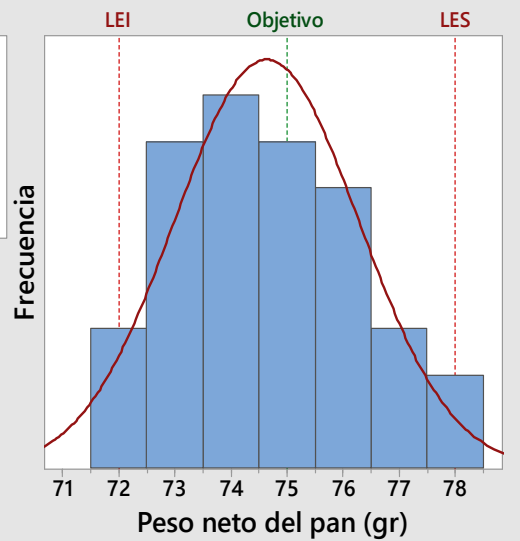

Capacidad largo plazo

Pp 0,61

PPL $\quad 0,54$

PPU 0,68

Ppk 0,54

Cpm $\quad 0,59$

Capacidad corto plazo

Cp $\quad 0,59$

CPL $\quad 0,52$

CPU $\quad 0,66$

Cpk 0,52

Peso neto del pan (gr)

\begin{tabular}{|lrrr|}
\hline & \multicolumn{2}{c}{ Rendimiento } \\
& Esperado Largo & Esperado Corto \\
& Observado & plazo & plazo \\
PPM $<$ LEI & 0,00 & 53957,96 & 59976,00 \\
PPM $>$ LES & 0,00 & 20298,88 & 23821,03 \\
PPM Total & 0,00 & 74256,84 & 83797,03 \\
\hline
\end{tabular}




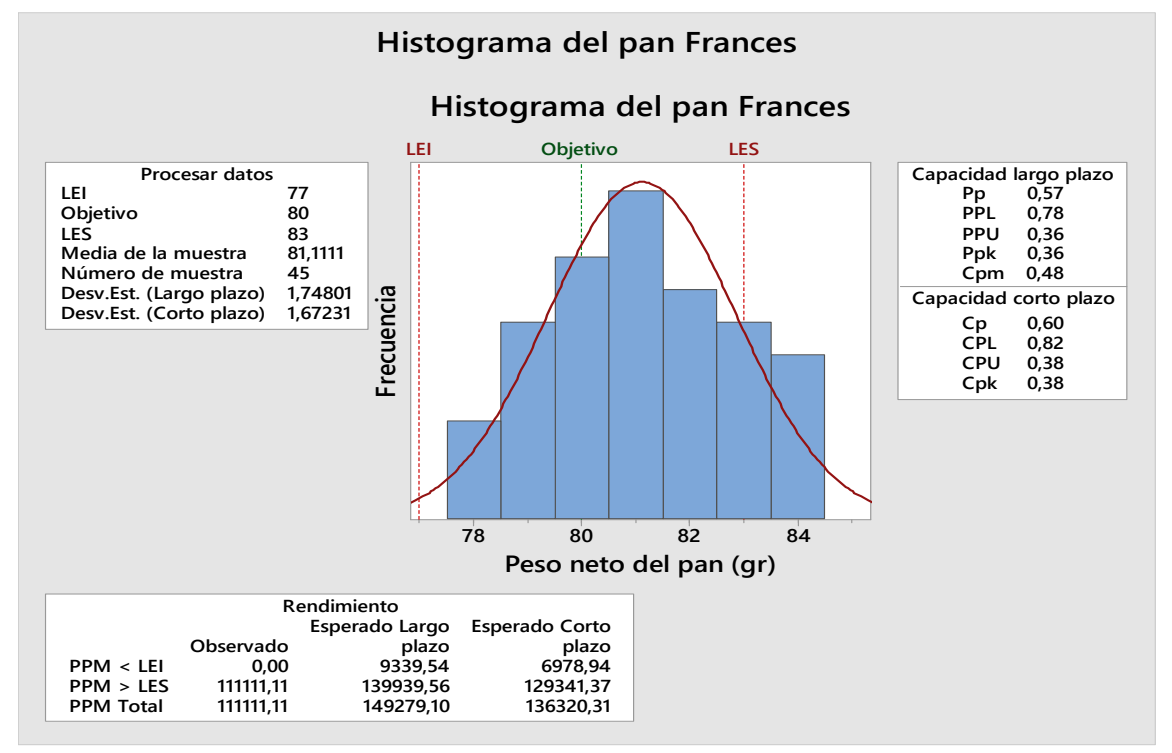

Nota. Fuente: construcción propia

Cabe destacar que, para evaluar el proceso, primero se debe determinar si los datos siguen una distribución normal, puesto que los análisis estadísticos que se desean aplicar se basan en el supuesto de que el comportamiento de los datos es normal. Por lo tanto, para verificar que los datos siguen una distribución normal se realizó una prueba de normalidad, utilizando el test de Kolmogorov (Pedrosa, Juarros, Robles, Basteiro, \& Eduardo, 2014), para ello se comprueba la hipótesis de que los datos son normales para un valor. El resultado del test se muestra en las figuras 3, 4 y 5 . 
Diseño de estrategias para el mejoramiento continuo de la calidad en el proceso de producción de productos de panadería de una microempresa

Figura 3

Prueba de normalidad del pan ocañero.

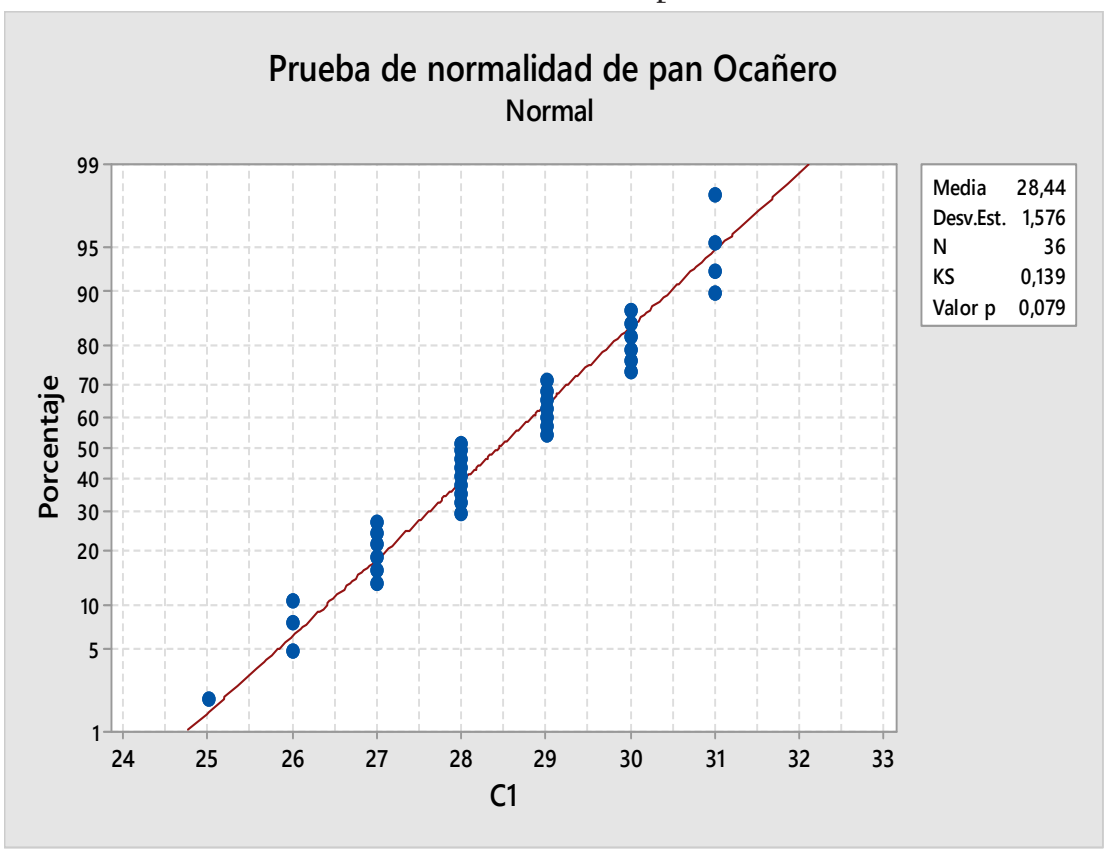

Nota. Fuente: construcción propia

Figura 4

Prueba de normalidad del pan integral.

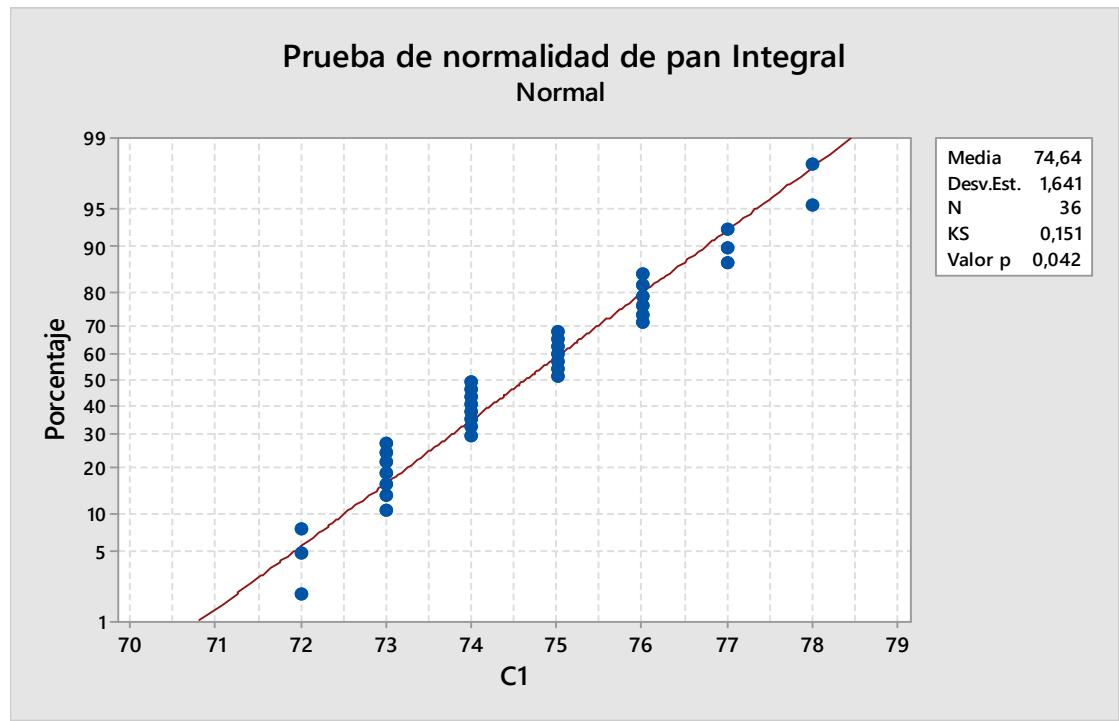

Nota. Fuente: construcción propia 
Figura 5

Prueba de normalidad del pan francés.

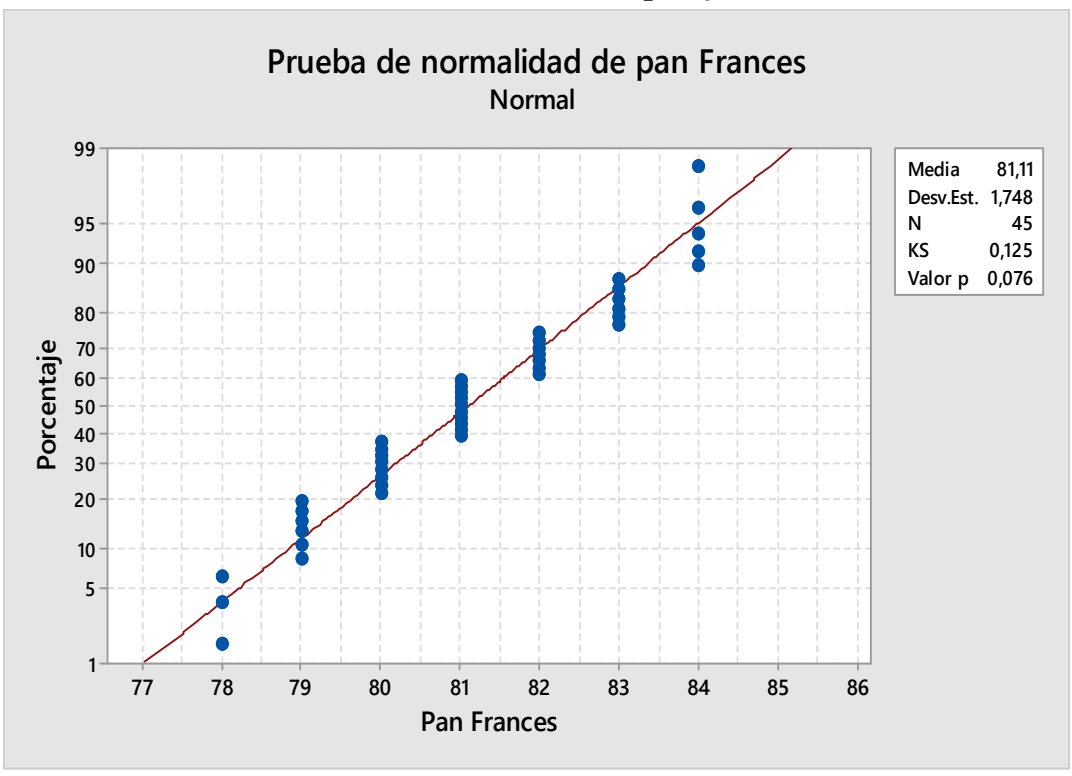

Nota. Fuente: construcción propia

El test para el pan ocañero, pan integral y pan francés arrojó un resultado de $0.139,0.151$ y 0.125 , respectivamente. Además, en la gráfica se observa que los valores de para el pan ocañero y pan francés son mayores a 0.05 , mientras que para el pan integral el valor de es igual a 0.042, por lo cual la hipótesis nula solo se acepta para el pan ocañero y el pan francés, y se infiere que los datos para estos dos panes se distribuyen de forma normal.

Luego de comprobar que los datos siguieran una distribución normal, se realizaron las cartas y para el pan ocañero y pan francés, con la finalidad de determinar si el proceso es estable o se encuentra bajo control estadístico. Estos gráficos permitirán detectar variaciones e identificar subgrupos que estén fuera de los límites de control o que siguen alguna tendencia. Las figuras 6 y 7 muestran los resultados obtenidos. 


\section{Figura 6}

Gráfico y para pan ocañero.

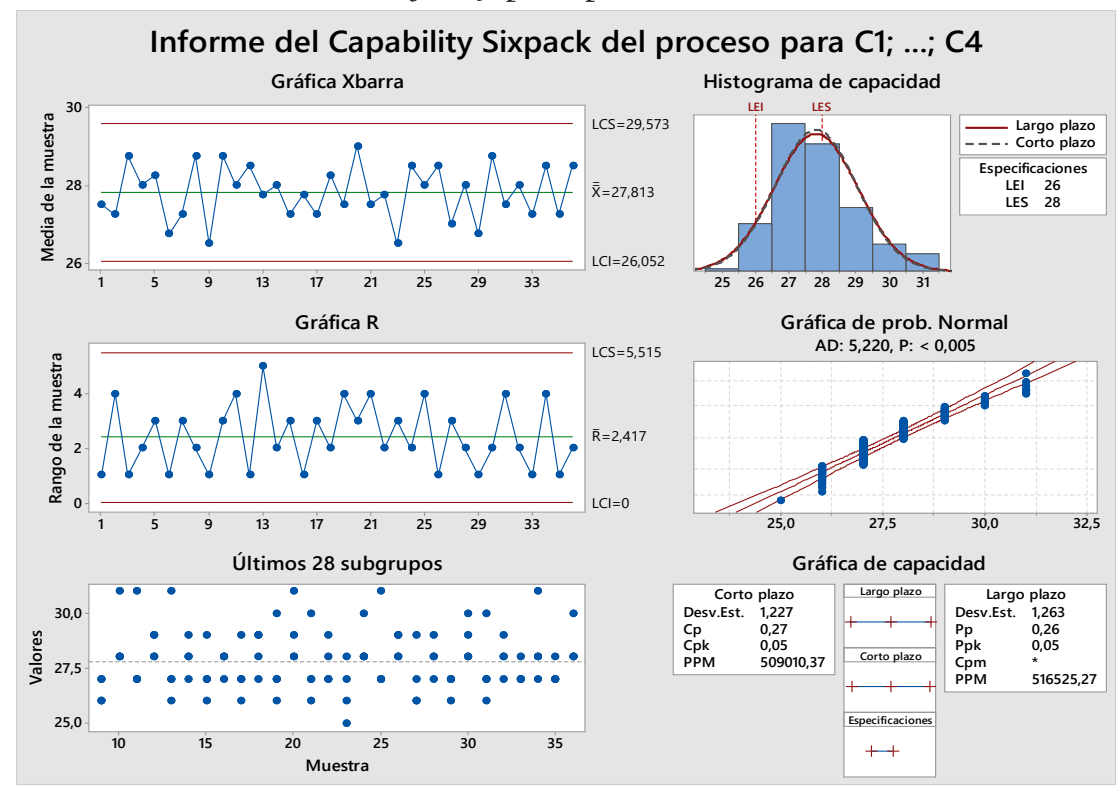

Nota. Fuente: construcción propia

Figura 7

Gráfico y para pan francés.

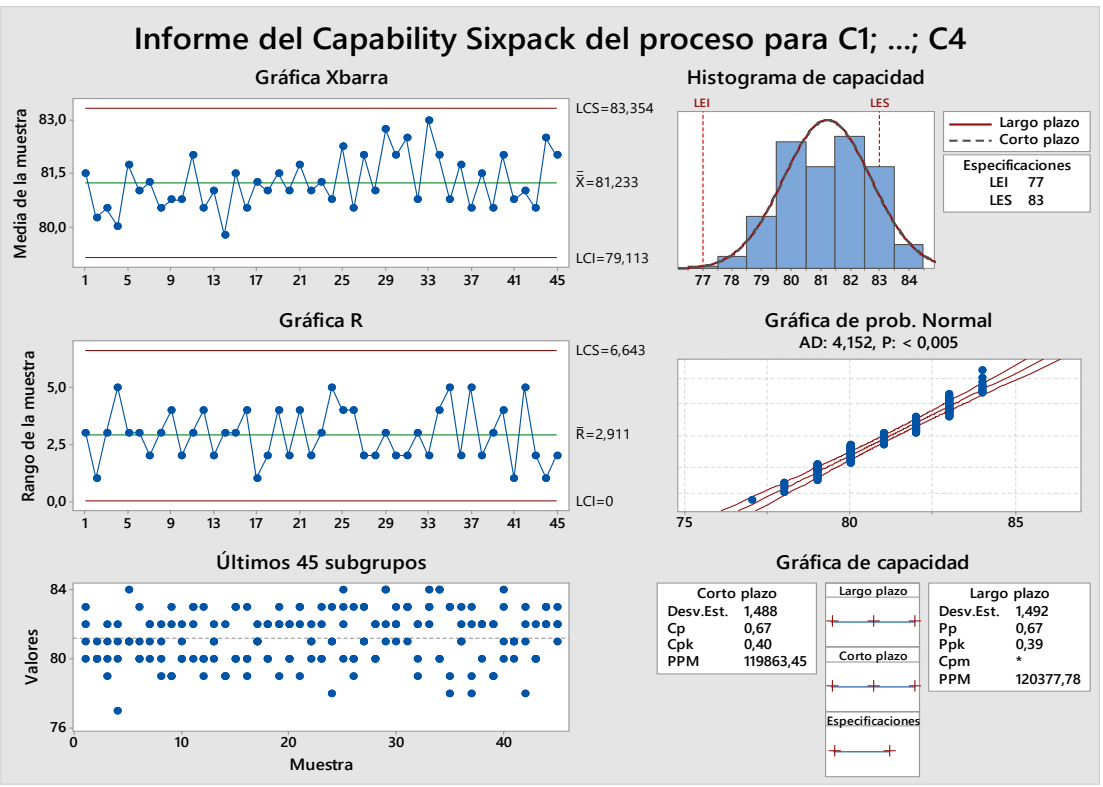

Nota. Fuente: construcción propia 
Los gráficos $\bar{X}$ y $R$, para el pan ocañero y francés, muestran que el proceso se encuentra bajo control estadístico, dado que en las gráficas de medias y rangos los resultados de las muestras están dentro de los límites de control. No obstante, en la gráfica $\bar{X}$ para el pan ocañero se observa una tendencia descendente del punto 10 al punto 15, a diferencia del pan francés, el cual no muestra tendencia. Por otra parte, la carta Apara el pan ocañero no muestra tendencia o racha, sin embargo, el pan francés muestra breves tendencias. En resumen, las cartas $\bar{X}_{\text {y }}$ Rmostraron que el proceso se encuentra bajo control, por lo tanto, no es necesario tomar medidas para cumplir con los límites de control, aunque se hace necesario investigar los subgrupos que tienen un comportamiento anormal.

Partiendo de que el proceso se encuentra bajo control, se procede a determinar si el proceso es capaz de cumplir con las especificaciones, mediante los índices de capacidad del proceso. En la siguiente tabla se muestra el resultado.

Tabla 3

Índice de capacidad del proceso.

\begin{tabular}{ccc}
\hline $\begin{array}{c}\text { Tipos de pan / medidas } \\
\text { de tendencia central }\end{array}$ & Pan ocañero & Pan francés \\
\hline $\boldsymbol{C}_{\boldsymbol{P}}$ & 0,57 & 0,71 \\
$\boldsymbol{C}_{\boldsymbol{P S}}$ & 0,62 & 0,39 \\
$\boldsymbol{C}_{\boldsymbol{P i}}$ & 0,51 & 1,03 \\
$\boldsymbol{C}_{\boldsymbol{P k}}$ & 0,51 & 0,39 \\
$\boldsymbol{K}$ & $9 \%$ & $45 \%$ \\
\hline
\end{tabular}

Nota. Fuente: construcción propia.

Podemos observar que el índice de capacidad $\boldsymbol{C}_{\boldsymbol{P}}$ y $\boldsymbol{C}_{\boldsymbol{P} \boldsymbol{k}}$ para el pan ocañero y francés es menor a 1 , lo cual indica que el proceso es de grado 3 , es decir, no tiene calidad. No obstante, se observa que el límite inferior de especificación del pan francés tiene una calidad parcialmente adecuada (grado 2), sin embargo, el límite de especificación superior no tiene calidad. Del anterior resultado se infiere que el proceso estadísticamente se encuentra bajo control, pero no es capaz de cumplir con las especificaciones. A continuación, se mostrará el porcentaje de productos que no cumple con las especificaciones. 


\section{Tabla 4}

Porcentaje de defectos de pan ocañero.

Tipos de pan $/ \%$ defectos

$P(x>30)$

$P(x<26)$

$P(x>30)+P(x<26)$
Pan ocañero

0,031

0,061

$9,2 \%$

Nota. Fuente: construcción propia.

Tabla 5

Porcentaje de defectos de pan francés.

\begin{tabular}{cc}
\hline $\begin{array}{c}\text { Tipos de pan / } \\
\% \text { defectos }\end{array}$ & Pan francés \\
\hline $\boldsymbol{P}(\boldsymbol{x}>\mathbf{8 3})$ & 0,122 \\
$\boldsymbol{P}(\boldsymbol{x}<\mathbf{7 7})$ & 0,001 \\
$\boldsymbol{P}(\boldsymbol{x}>\mathbf{3 0})+\boldsymbol{P}(\boldsymbol{x}<\mathbf{2 6})$ & $12 \%$ \\
\hline
\end{tabular}

Nota. Fuente: construcción propia.

Las tablas 4 y 5 muestran que el porcentaje de productos defectuosos para el pan ocañero es de 9,2\% y para el pan francés es de $12 \%$.

Con la anterior información se realizó el análisis de causa y efecto en el proceso de la fabricación de los tipos de panes defectuosos, para así poder encontrar la causa que genera esta inconformidad con las especificaciones y, de esta manera, poder ajustar los procesos. A continuación, se muestra el diagrama de causa-efecto con el cual se organizan, de forma gráfica, las posibles causas del problema específico de la producción. 
Figura 8

Diagrama de causa-efecto.

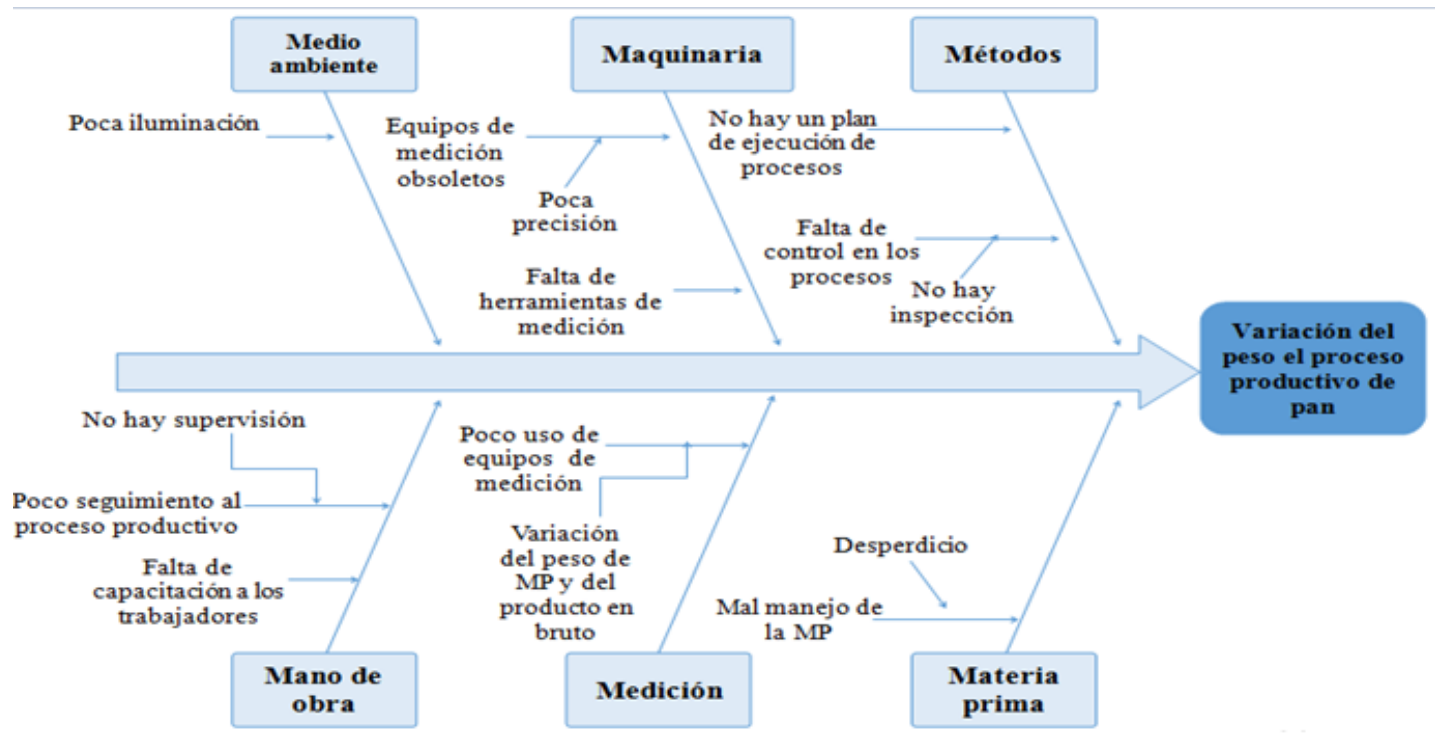

Nota. Fuente: construcción propia.

Con la aplicación de esta herramienta, se identificaron los factores que están provocando que el proceso no marche de la mejor manera; a su vez, se encontraron las diferentes causas y efectos que pueden afectar el proceso, estudiando estos factores bajo el análisis de las $6 \mathrm{M}$; dicho análisis evalúa los factores de medio ambiente, métodos, materiales, mano de obra, materia prima y mediciones (Andrade, Del Río, \& Alvear, 2019).

El principal problema presentado consiste en la mala medición del peso en gramos del pan en bruto, para lo cual se parte de una secuencia de causas las cuales están descritas en el gráfico. Partiendo de una lluvia de ideas para determinar tales causas, este diagrama nos muestra las causas particulares que pueden estar ocasionando que el proceso no marche de buena manera y, asimismo, el efecto que provoca que el pan no cumpla con los estándares de calidad adecuados. 
Tabla 5

Matriz AMEF.

\begin{tabular}{|c|c|c|c|c|c|c|c|c|c|c|}
\hline $\begin{array}{l}\text { Pasos Clave } \\
\text { del Proceso }\end{array}$ & Entradas & $\begin{array}{c}\text { Modos } \\
\text { de Falla } \\
\text { Potenciales }\end{array}$ & $\begin{array}{c}\text { Efectos de Fallas } \\
\text { Potenciales }\end{array}$ & SEV & $\begin{array}{c}\text { Causas } \\
\text { Potenciales }\end{array}$ & OCU & $\begin{array}{l}\text { Controles de } \\
\text { Ocurrencia }\end{array}$ & DET & PR & $\begin{array}{c}\text { Acciones } \\
\text { Recomendadas }\end{array}$ \\
\hline $\begin{array}{l}\text { Pesado de la } \\
\text { materia prima }\end{array}$ & $\begin{array}{l}\text { Cantidades de } \\
\text { materia prima }\end{array}$ & $\begin{array}{l}\text { Mala } \\
\text { administración } \\
\text { de la materia } \\
\text { prima }\end{array}$ & $\begin{array}{l}\text { Variación del peso } \\
\text { en la materia prima } \\
\text { utilizada }\end{array}$ & 7 & $\begin{array}{l}\text { Uso de } \\
\text { herramientas } \\
\text { poco precisas }\end{array}$ & 8 & No hay & 9 & 504 & $\begin{array}{l}\text { Adquirir mejores } \\
\text { herramientas para el } \\
\text { pesado de la MP }\end{array}$ \\
\hline $\begin{array}{l}\text { Mezclado y } \\
\text { paso por rodillo } \\
\text { eléctrico }\end{array}$ & $\begin{array}{l}\text { Tiempo de } \\
\text { mezclado }\end{array}$ & $\begin{array}{l}\text { Poca } \\
\text { homogeneidad } \\
\text { en la masa }\end{array}$ & $\begin{array}{l}\text { Textura no } \\
\text { apropiada }\end{array}$ & 6 & $\begin{array}{l}\text { Falta de } \\
\text { experiencia } \\
\text { trabajando con el } \\
\text { rodillo eléctrico }\end{array}$ & 2 & No hay & 2 & 24 & Supervisar el proceso \\
\hline Mezclado manual & Amasado & $\begin{array}{l}\text { Poca } \\
\text { homogeneidad } \\
\text { en la masa }\end{array}$ & $\begin{array}{l}\text { Textura no } \\
\text { apropiada }\end{array}$ & 7 & $\begin{array}{l}\text { Poco tiempo de } \\
\text { amasado manual }\end{array}$ & 2 & No hay & 3 & 42 & $\begin{array}{l}\text { Seguimiento y supervisión } \\
\text { del proceso }\end{array}$ \\
\hline $\begin{array}{l}\text { Reposo de la } \\
\text { masa }\end{array}$ & $\begin{array}{l}\text { Tiempo de } \\
\text { fermentado }\end{array}$ & $\begin{array}{l}\text { Poco tiempo de } \\
\text { reposo }\end{array}$ & $\begin{array}{l}\text { Fallas en el } \\
\text { crecimiento de la } \\
\text { masa }\end{array}$ & 8 & $\begin{array}{l}\text { Aceleración } \\
\text { en el proceso } \\
\text { productivo }\end{array}$ & 2 & No hay & 3 & 48 & $\begin{array}{l}\text { Tener un control del } \\
\text { crecimiento de la masa }\end{array}$ \\
\hline $\begin{array}{l}\text { Corte y pesado de } \\
\text { la masa }\end{array}$ & $\begin{array}{l}\text { Métodos de } \\
\text { corte y pesado } \\
\text { de la masa }\end{array}$ & $\begin{array}{l}\text { Pequeñas } \\
\text { variaciones } \\
\text { del peso de las } \\
\text { porciones de la } \\
\text { masa }\end{array}$ & $\begin{array}{l}\text { Volumen y tamaño } \\
\text { variado }\end{array}$ & 8 & $\begin{array}{l}\text { Falta de } \\
\text { herramientas de } \\
\text { medición }\end{array}$ & 8 & No hay & 8 & 512 & $\begin{array}{l}\text { Adquirir equipos de } \\
\text { medición para el peso del } \\
\text { producto bruto }\end{array}$ \\
\hline Moldeado & $\begin{array}{l}\text { Método del } \\
\text { moldeado }\end{array}$ & $\begin{array}{l}\text { Variación en } \\
\text { el tamaño del } \\
\text { producto bruto }\end{array}$ & $\begin{array}{l}\text { Pequeñas } \\
\text { deformaciones }\end{array}$ & 7 & $\begin{array}{l}\text { Personal poco } \\
\text { capacitado }\end{array}$ & 2 & No hay & 3 & 42 & $\begin{array}{l}\text { Capacitar constantemente } \\
\text { a los empleados en } \\
\text { técnicas de repostería }\end{array}$ \\
\hline $\begin{array}{l}\text { Reposo del } \\
\text { producto en } \\
\text { bruto }\end{array}$ & $\begin{array}{l}\text { Tiempo de } \\
\text { fermentación }\end{array}$ & $\begin{array}{l}\text { Poco tiempo de } \\
\text { reposo }\end{array}$ & $\begin{array}{l}\text { Fallas en el } \\
\text { crecimiento del } \\
\text { pan en bruto }\end{array}$ & 8 & $\begin{array}{l}\text { Aceleración } \\
\text { en el proceso } \\
\text { productivo }\end{array}$ & 2 & $\begin{array}{l}\text { Supervisión } \\
\text { del } \\
\text { crecimiento } \\
\text { y estimación } \\
\text { de tiempos de } \\
\text { reposo }\end{array}$ & 2 & 32 & $\begin{array}{l}\text { Llevar estandarizados los } \\
\text { tiempos adecuados para el } \\
\text { crecimiento del pan }\end{array}$ \\
\hline Horneado & $\begin{array}{l}\text { Tiempo de } \\
\text { horneado }\end{array}$ & $\begin{array}{l}\text { Falta de } \\
\text { atención en } \\
\text { el tiempo de } \\
\text { horneado }\end{array}$ & $\begin{array}{l}\text { Pan blando, pan } \\
\text { duro, color no } \\
\text { adecuado }\end{array}$ & 9 & $\begin{array}{l}\text { No } \\
\text { estandarización } \\
\text { de los tiempos de } \\
\text { horneado }\end{array}$ & 2 & & 2 & 36 & $\begin{array}{l}\text { Mejorar un poco los } \\
\text { estándares de tiempo de } \\
\text { horneado }\end{array}$ \\
\hline
\end{tabular}

Nota. Fuente: construcción propia

La matriz de análisis de modo y efecto de falla permite identificar el grado de criticidad de los procesos, basado en su severidad, ocurrencia y detección. Tales medidas pueden influir de madera significativa en la calidad del pan, la cual puede ser percibida por el cliente. En la matriz AMEF se identificaron como fallas potenciales los siguientes aspectos: cantidad de MP utilizada al inicio del proceso, método de corte y pesado de la masa. Al analizar el resultado obtenido en el NPR (Numero
Prioritario de Riesgo), se dice que los efectos que mayor afectan el proceso son los mencionados anteriormente. Es necesario dar una pronta solución a estos problemas ya que según el índice NPR presentan un riesgo de falla alto, lo cual no es muy bueno si se quiere obtener una buena calidad. 


\section{Conclusiones}

La observación de la variación del tamaño de cada pan, teniendo en cuenta el valor nominal y la tolerancia establecida en la panificadora, por medio de las herramientas de control de calidad como histogramas, pruebas de normalidad, cartas de control, entre otras, permitió identificar los principales problemas de calidad de la empresa y diseñar estrategias para la eliminación de las causas primordiales de los mismos. Estas estrategias fueron: la estandarización del proceso de medición de la cantidad de ingredientes a utilizar, el establecimiento de programas de capacitación y la adquisición de herramientas de corte de mayor precisión.

Por otra parte, la aplicación del diagrama de causa- efecto y la implementación de la matriz AMEF permitieron conocer en qué está fallando el proceso e identificar específicamente cuáles son las causas de fallo más críticos, lo cual influyó en la priorización de los mismos para darle una pronta solución al problema.

\section{Referencias}

Andrade, A., Del Río, C., \& Alvear, D. (2019). Estudio de Tiempos y Movimientos para Incrementar la Eficiencia en una Empresa de Producción de Calzado. SCielo.

Cartín, A. R., Villarreal, A. T., \& Morera, A. (2014). Implementación del análisis de riesgo en la industria alimentaria mediante la metodología AMEF: enfoque práctico y conceptual. SCielo.

Gutiérrez, H. (2009). CONTROL ESTADÍSTICO DE CALIDAD Y SEIS SIGMA. México: Mc Graw Hill.

Mosquera, J. D., Artamonova, I., \& Mosquera, J. C. (2013). Diagnóstico del proceso de inspección mediante índices de capacidad. SCielo.

Pedrosa , I., Juarros, J., Robles, A., Basteiro, J., \& Eduardo, G. (2014). Pruebas de bondad de ajuste en distribuciones. Scielo.

Pértegas Díaz S., P. F. (2001). La distribución normal. Unidad de Epidemiología Clínica y Bioestadística, 1.

Rivera García, D. (2011). CARTAS DE CONTROL PARA DATOS FUNCIONALES. 
Diseño de estrategias para el mejoramiento continuo de la calidad en el proceso de producción de productos de panadería de una microempresa

Rodríguez, A. I., \& Franco, J. R. (2009). Control estadístico de la calidad de un servicio mediante Gráficas X y R. Universidad Autónoma Metropolitana Xochimilco, 156.

Rojas, A. R.-F. (2006). CONTROL ESTADÍSTICO DE PROCESOS. Madrid : universidad pontificia icai.

Rubio, J., Angeles, J., Soto, Y., \& Toma, J. (1995). Procesamiento Estadístico de Datos con Minitaby Harvard Graphics. Lima Perú. 